\title{
Oropharyngeal Swab Specimen
}

National Cancer Institute

\section{Source}

National Cancer Institute. Oropharyngeal Swab Specimen. NCI Thesaurus. Code C155835.

A biospecimen collected from the oropharynx by swabbing. 